\title{
Improving Worker Safety for Handling Nanomaterials at the Benchtop.
}

\author{
Byron D. Gates ${ }^{1 *}$, Kelly Cadieux ${ }^{1}$, Matt Bilton ${ }^{1}$, Iris Guo ${ }^{1}$, Tania Hildago Castillo ${ }^{1}$, Taeeun Chung ${ }^{1}$, Tony
} Ngo $^{1}$, and Clifton Bright Davies ${ }^{1}$

1. Department of Chemistry, Simon Fraser University, Burnaby, BC, Canada; (*email: bgates@sfu.ca)

Nanomaterials have become common place in the laboratory, as well as in product development. Examples include new formulations of materials with enhanced antimicrobial properties, increased efficiency of energy conversion (whether for catalysis or for photochemical processes), and a utility in creating new formulations for delivery and release of drugs. There are, however, a number of unknowns surrounding nanomaterials. We are just beginning to understand the various pathways that nanomaterials take within biological and ecological systems as we seek to understand their ultimate fate when encountered in either type of system. At the forefront of this challenge is, however, to better understand the safety of workers who are initially creating, processing or otherwise coming into contact with these materials. Further science is needed to understand the potential for exposure to nanomaterials in the workplace environment.

One of the benefits for working with nanomaterials is their small size, but this also presents a challenge for assessing their presence in the workplace environment. ${ }^{1}$ Due to their small size, nanomaterials can often go unnoticed by workers unless, for example, they are accumulated in relatively large quantities. Protocols are being developed around the world to protect workers from undue exposure to these materials. For example, a number of studies are pursuing the testing, development and implementation of appropriate personal protective equipment (PPE) for workers handling and otherwise processing nanomaterials. Progress has also been made to improve signage specific to the use and storage of nanomaterials in the workplace. Other areas of science still need further work to understand how to safely handle these materials.

Understanding the extent of and methods to deal with potential spills, which may go overlooked in the laboratory, is the focus of our studies. This forensic investigation seeks to identify and monitor nanomaterial spills in a simulated workplace environment. ${ }^{1,2}$ These studies aim to develop procedures to refine current laboratory practices, as necessary, and to identify methods for dealing with potential spills. A variety of analytical techniques have been utilized to identify and track simulated spills containing nanomaterials (e.g., Figure 1). These techniques include the use of fluorescence microscopy, electron microscopy, and a variety of elemental analysis methods (e.g., inductively coupled plasma mass spectrometry and energy dispersive X-ray spectroscopy). This analysis and tracking of the simulated spills is further extended to monitoring our ability to clean-up these spills. There are a variety of methods we are developing to remediate spills containing nanomaterials. These methods are followed for their efficiency, or ability to efficiently remove the nanomaterials from simulated spills on sections of countertop commonly found in the laboratory (Figure 2). ${ }^{1,2}$ A variety of methods will be introduced and compared through this presentation. The methods were tested against variables that include the size and composition of the nanomaterials, and surfactants present in the samples.

In summary, this presentation will review progress in establishing analytical techniques that have been tested to monitor the spill of nanomaterials in the workplace, along with a discussion of the next steps to establish the effective means of cleaning up after potential spills of nanomaterials. 
[1] BD Gates et al, "Detecting, Handling and Controlling Nanoparticle Contamination in the Workplace, Final Report for WorkSafeBC and Workers' Compensation Board of Nova Scotia”, Project number: RS2010-IG40, (2013).

[2] J Zhou, M.Sc. Thesis, Simon Fraser University (2015).

[3] We acknowledge funding support in part from WorkSafeBC through the Innovation at Work program, the Natural Sciences and Engineering Research Council (NSERC) of Canada, the Canada Research Chairs Program (B.D. Gates), and CMC Microsystems through the MNT Financial Assistance program that facilitated access to materials characterization services. This work made use of 4D LABS shared facilities supported by the Canada Foundation for Innovation (CFI), British Columbia Knowledge Development Fund (BCKDF), Western Economic Diversification Canada, and Simon Fraser University.
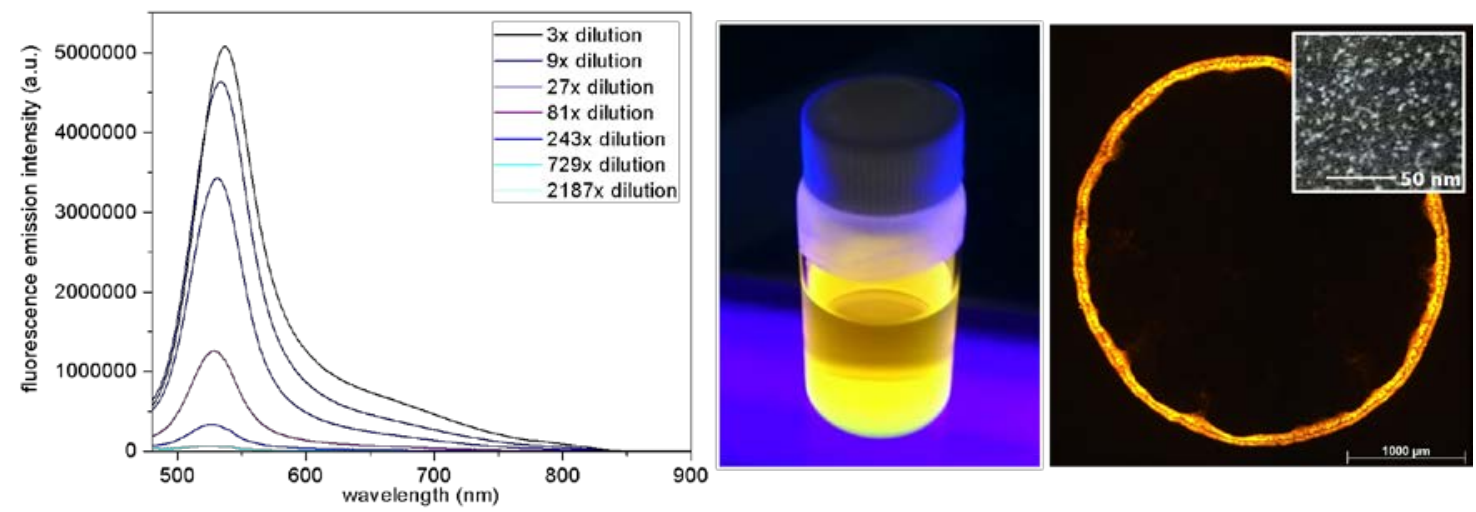

Figure 1. Quantum dots prepared to simulate spills that could happen in the workplace. These nanomaterials can be traced in these simulated tests using their fluorescent properties as confirmed by fluorescence emission spectroscopy, depicted in the fluorescent image of a vial of these materials, and demonstrated by fluorescence microscopy in a simulated spill on a section of countertop. Inset is a transmission electron microscopy image of the as-prepared quantum dots.


Figure 2. A comparison of the efficiency of two methods for remediating spills of nanomaterials from a simulated spill on sections of countertop commonly found in the laboratory, and a scanning electron microscopy image of a spill of silver nanoparticles on such a section of countertop. The SEM image suggests the presence of both finely dispersed and aggregates of nanoparticles. 